\title{
Article
}

\section{Generalized fractional differential ring}

\author{
Zeinab Toghani ${ }^{1}$ and Luis Gaggero-Sager ${ }^{1, *}$ \\ 1 Queen Mary University of London, Universidad Autónoma del Estado de Morelos(CIICAp), London. \\ * Correspondence: lgaggero@uaem.mx
}

Academic Editor: Dumitru Vieru

Received: 4 March 2021; Accepted: 17 July 2021; Published: 4 September 2021.

\begin{abstract}
There are many possible definitions of derivatives, here we present some and present one that we have called generalized that allows us to put some of the others as a particular case of this but, what interests us is to determine that there is an infinite number of possible definitions of fractional derivatives, all are correct as differential operators each of them must be properly defined its algebra. We introduce a generalized version of fractional derivative that extends the existing ones in the literature. To those extensions it is associated a differentiable operator and a differential ring and applications that shows the advantages of the generalization. We also review the different definitions of fractional derivatives and it is shown how the generalized version contains the previous ones as a particular cases.
\end{abstract}

Keywords: Generalized fractional derivative; Fractional differential ring; Fractional differential equation.

MSC: Missing

\section{Introduction and Preliminaries}

$\mathbf{F}$ ractional derivative was defined for responding to a question 'what does it mean $\frac{d^{\alpha} f}{d t^{\alpha}}$ if $\alpha=\frac{1}{2}$ ' in 1695 . Following that, finding the right definition of fractional derivative has attracted significant attention of researcher and in the last few years it has seen significantly progress in mathematical and non-mathematical journals (see [1-10]). In fact, there are articles which in few months have gained hundreds of citations. In particular in past three years several definitions of fractional derivative have been proposed (see [3,4,10-23]). Since some of previous definitions do not satisfy the classical formulas of the usual derivative, it has been proposed an ad hoc algebra associated to each definition. To unify that diversity, we propose a version of fractional derivative that has the advantages that generalized the already existing in the literature and where the different algebras are unified under the notion of fractional differential ring.

The present paper is organized as follows: In the Section 2 we give the previous definitions of fractional derivative and our generalized fractional derivative (GFD) definition, in the Section 3 we introduce a fractional differential ring, in the Section 4 we give some result of GFD, in the Section 5 we give a definition of partial fractional differential derivative, in the Section 6 we give a definition of GFD when $\alpha \in(n, n+1]$.

\section{Fractional derivative}

Let $\alpha \in(0,1]$ be a fractional number, we want to give a definition of generalized fractional derivative of order $\alpha$ for a differentiable function $f$. We denote $\alpha$-th derivative of $f$ by $D^{\alpha}(f)$ and we denote the first derivative of $f$ by $D(f)$.

We begin the present section listing previous definition of fractional derivative; later we present our proposal of generalized one showing how it contains the once already described. We finish the section providing some examples.

1. The Caputo fractional derivative was defined by Michele Caputo in [24]:

$$
D^{\alpha}(f)=\frac{1}{\Gamma(1-\alpha)} \int_{a}^{t} \frac{f^{\prime}(x)}{(t-x)^{\alpha}} d x .
$$


2. The conformable fractional derivative was defined by Khalil, Al Horani, Yousef and Sababheh in [11]:

$$
D^{\alpha} f(t)=\lim _{\varepsilon \rightarrow 0} \frac{f\left(t+t^{1-\alpha} \varepsilon\right)-f(t)}{\varepsilon} .
$$

3. The conformable fractional derivative was defined by Anderson and Ulness in [25]:

$$
D^{\alpha} f(t)=(1-\alpha)|t|^{\alpha} f(t)+\alpha|t|^{1-\alpha} D f
$$

4. The fractional derivative was defined by Udita N.Katugampola in [17]:

$$
D^{\alpha} f(t)=\lim _{\varepsilon \rightarrow 0} \frac{f\left(t e^{\varepsilon t^{-\alpha}}\right)-f(t)}{\varepsilon} .
$$

5. The fractional derivative was defined by Guebbai and Ghiat in [20] for an increasing and positive function $f$ :

$$
D^{\alpha} f(t)=\lim _{\varepsilon \rightarrow 0}\left(\frac{f\left(t+f(t)^{\frac{1-\alpha}{\alpha}} \varepsilon\right)-f(t)}{\varepsilon}\right)^{\alpha} .
$$

6. The conformable ratio derivative was defined by Camrud in [14] for a function $f(t) \geq 0$ with $D f(t) \geq 0$ :

$$
D^{\alpha} f(t)=\lim _{\varepsilon \rightarrow 0} f(t)^{1-\alpha}\left(\frac{f(t+\varepsilon)-f(t)}{\varepsilon}\right)^{\alpha} .
$$

From all these definitions, we propose a definition that unifies almost all of them.

Definition 1. Given a differentiable function $f:[0, \infty) \rightarrow \mathbb{R}$, the generalized fractional derivative(GFD) for $\alpha \in(0,1]$ at point $t$ is defined by:

$$
D^{\alpha} f(t)=\lim _{\mathcal{E} \rightarrow 0} \frac{f\left(t+w_{t, \alpha} t^{1-\alpha} \varepsilon\right)-f(t)}{\varepsilon}
$$

where $w_{t, \alpha}$ is a function that may depend on $\alpha$ and $t$.

Remark 1. As a consequence of Definition 1 we can see

$$
D^{\alpha} f(t)=w_{t, \alpha} t^{1-\alpha} D f(t)
$$

Definition 2. A differentiable function $f:[0, \infty) \rightarrow \mathbb{R}$ is said to be $\alpha$-generalized fractional differentiable function over $[0, \infty)$ if it exists $D^{\alpha}(f)(t)$ for all $t \in[0, \infty)$ for $\alpha \in(0,1]$.

We denote $C^{\alpha}[0, \infty)$ the set of $\alpha$-generalized differentiable functions with real values in the interval $[0, \infty)$ in variable $t$. The set $\left(C^{\alpha}[0, \infty),+,.\right)$ is a ring. In the following we want to see the relation between GFD and the others definitions:

1. The fractional derivative of Khalil, Al Horani, Yousef and Sababheh in [11] is a particular case of GFD where $w_{t, \alpha}=1$.

2. The fractional derivative of Anderson and Ulness in [25] is a particular case of GFD where

$$
w_{t, \alpha}=\frac{(1-\alpha) t^{\alpha} f(t)+\alpha t^{1-\alpha} D f}{\alpha t^{1-\alpha}} .
$$

In this fractional derivative $w_{t, \alpha}$ depends on $\alpha$ and $t$.

3. The fractional derivatives of Guebbai and Ghiat in [20] and Camrud in [14] are particular cases of GFD where $w_{t, \alpha}=\left(\frac{t D f}{f}\right)^{\alpha-1}$.

We are particularly interested in discussing GFD where $w_{t, \alpha}=g(t, \alpha) \tau^{\alpha-1}$ such that $g:[0, \infty) \times(0,1] \rightarrow \mathbb{R}$ is a function and $\tau$ is the characteristic of system with the properties

$$
w_{t, \alpha}=1 \text { if and only if } \alpha=1 \text {. }
$$


If the system is periodic with period $T$, then we have $\tau=T$. In the quantum systems $\tau$ is the Bohr radius and in astronomy $\tau$ is the light year. The characteristic of system $\tau$ depends on the systems and the derivative. If $t$ is time, $\tau$ is time too. If $t$ is space, $\tau$ is space too. In fact the unit of $t$ is $\tau$, i.e., $t=c \tau$ where $c$ is a constant. In the general $\tau=1$.

Example 1. Let $\alpha, \beta \in(0,1]$. Let $f, h$ be two functions in $C^{\alpha}[0, \infty)$. We suppose $w_{t, \alpha}=g(t, \alpha) \tau^{\alpha-1}$ with $\tau=1$.

1. If $g(t, \alpha)$ is a function with $g(0,0)=0$, then $\lim _{\alpha \rightarrow 0} D^{\alpha}(f)=0$.

2. If $g(t, \alpha)=\alpha$, we have the chain rule

$$
D^{\alpha}(f \circ h)=\frac{t^{\alpha-1}}{\alpha} D^{\alpha}(f(h)) D^{\alpha}(h) .
$$

Example 2. We want to present the corresponding figure to the generalized fractional derivatives for $\alpha=\frac{3}{4}$ for a trigonometric, using all the fractional derivative definitions that we have already mentioned in this article. It can be seen from all the figures that in principle these definitions do not find a reason to discard them. That is, they have a fairly reasonable behavior. We consider $f(t)=\sin (2 t)$, the graph of $f$ can be seen in Figure 1 .

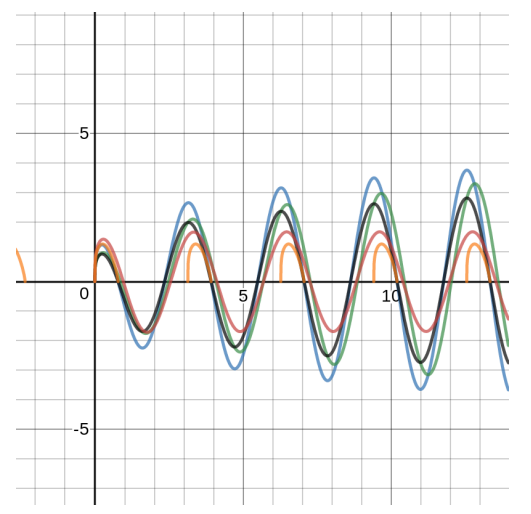

Figure 1. red:Caputo, green:Khalil et al., blue:Anderson et al., orange:Guebbai et al., black:GFD when $w_{t, \alpha}=\alpha$.

\section{Generalized fractional differential ring}

In this section we want to stress out that instead of defining a new derivative, we focus on the notion of differentiable operator and the ring that it carries with.

Definition 3. Let $R$ be a commutative ring with unity. A derivation on $R$ is a map $d: R \rightarrow R$ that satisfies $d(a+b)=d(a)+d(b)$ and $d(a b)=d(a) b+a d(b), \forall a, b \in R$. The pair $(R, d)$ is called a differential ring (see [26]).

Theorem 1. Let $\alpha \in(0,1]$, then the ring $C^{\alpha}[0, \infty)$ with operator $D^{\alpha}: C^{\alpha}[0, \infty) \rightarrow C^{\alpha}[0, \infty)$ is a differential ring.

Proof. Since $C^{\alpha}[0, \infty)$ is a commutative ring with unity $f(t)=1$ and the derivation $D^{\alpha}$ for $\alpha \in(0,1]$ satisfies following properties from Remark 1

1. $D^{\alpha}\left(a f_{1}+b f_{2}\right)=a D^{\alpha}\left(f_{1}\right)+b D^{\alpha}\left(f_{2}\right), \forall f_{1}, f_{2} \in C^{\alpha}[0, \infty), \forall a, b \in \mathbb{R}$,

2. $D^{\alpha}\left(f_{1} f_{2}\right)=f_{1} D^{\alpha}\left(f_{2}\right)+f_{2} D^{\alpha}\left(f_{1}\right), \forall f_{1}, f_{2} \in C^{\alpha}[0, \infty)$.

Let $\alpha \in(0,1]$ be a fractional number, and $f_{1}, f_{2} \in C^{\alpha}[0, \infty)$ be two functions, then GFD has the following properties:

1. $D^{\alpha}\left(\frac{f_{1}}{f_{2}}\right)=\frac{f_{2} D^{\alpha} f_{1}-f_{1} D^{\alpha} f_{2}}{f_{2}^{2}}$.

2. $D^{\alpha}\left(f_{1} \circ f_{2}\right)=\frac{t^{\alpha-1}}{w_{t, \alpha}} D^{\alpha}\left(f_{1}\left(f_{2}\right)\right) D^{\alpha}\left(f_{2}\right)$.

3. $D^{\alpha+\beta}\left(f_{1}\right)=\frac{w_{t, \alpha} w_{t, \beta} t}{w_{t, \alpha+\beta}} D^{\alpha} D^{\beta}\left(f_{1}\right)$. 
It is easy to see these properties from Remark 1 that if $w_{t, \alpha}=t^{1-\alpha}$ we have the equality

$$
D^{\alpha}\left(f_{1} \circ f_{2}\right)=D^{\alpha}\left(f_{1}\left(f_{2}\right)\right) D^{\alpha}\left(f_{2}\right)
$$

If $\frac{w_{t, \alpha+\beta}}{w_{t, \alpha} w_{t, \beta}}=t$ we have the equality

$$
D^{\alpha+\beta}\left(f_{1}\right)=D^{\alpha} D^{\beta}\left(f_{1}\right), \forall \alpha, \beta \in(0,1] .
$$

Parts 4 and 5 of the properties imply that we can create function spaces with different algebras using different expressions for $w_{t, \alpha}$.

By considering previous properties of GFD we called $C^{\alpha}[0, \infty)$ a $w_{t, \alpha}$-generalized fractional differential ring of functions and we denote it by $\left(C^{\alpha}[0, \infty), D^{\alpha}, w_{t, \alpha}\right)$. Let $I \subset C^{\alpha}[0, \infty)$ be an ideal. If $D^{\alpha}(I) \subset I$ then the ideal $I$ is called a $w_{t, \alpha}$-generalized fractional differential ideal. By using the previous properties we can see the following result:

Theorem 2. Let $\alpha \in(0,1]$, then, associated to any $\alpha$ and any $w_{t, \alpha}$ there exists a fractional differential ring.

\section{Some results of Generalized Fractional Derivative}

Let $\alpha \in(0,1]$ be a fractional number and $t \in[0, \infty)$ then GFD has the following properties:

1. $D^{\alpha}\left(\frac{t^{\alpha}}{\alpha w w_{t, \alpha}}\right)=1$,

2. $D^{\alpha}\left(\sin \left(\frac{t^{\alpha}}{\alpha w_{t, \alpha}}\right)\right)=\cos \left(\frac{t^{\alpha}}{\alpha w_{t, \alpha}}\right)$,

3. $D^{\alpha}\left(\cos \left(\frac{t^{\alpha}, \alpha}{\alpha w_{t, \alpha}}\right)\right)=-\sin \left(\frac{t^{\alpha}}{\alpha w_{t, \alpha}}\right)$,

4. $D^{\alpha}\left(e^{\left(\frac{t^{\alpha}}{\alpha w t_{, \alpha}}\right)}\right)=e^{\left(\frac{t^{\alpha}}{\alpha w_{t, \alpha}}\right)}$.

Theorem 3. (Rolle's Theorem for $\alpha$-Generalized Fractional Differentiable Functions)

Let $a>0$ and $f:[a, b] \rightarrow \mathbb{R}$ be a function with the properties that

1. $f$ is continuous on $[a, b]$,

2. $f$ is $\alpha$-generalized fractional differentiable on $(a, b)$ for some $\alpha \in(0,1]$,

3. $f(a)=f(b)$.

Then, there exist $c \in(a, b)$ such that $D^{\alpha} f(c)=0$.

Proof. Since $f$ is continuous on $[a, b]$ and $f(a)=f(b)$, then the function $f$ has a local extreme in a point $c \in(a, b)$ and

$$
D^{\alpha} f(c)=\lim _{\varepsilon \rightarrow 0^{+}} \frac{f\left(c+w_{t, \alpha} c^{1-\alpha} \varepsilon\right)-f(c)}{\varepsilon}=\lim _{\varepsilon \rightarrow 0^{-}} \frac{f\left(c+w_{t, \alpha} c^{1-\alpha} \varepsilon\right)-f(c)}{\varepsilon} .
$$

But two limits have different signs, so $D^{\alpha} f(c)=0$.

Theorem 4. (Mean Value Theorem for $\alpha$-Generalized Fractional Differentiable Functions) Let $a>0$ and $f:[a, b] \rightarrow \mathbb{R}$ be a function with the properties that

1. $f$ is continuous on $[a, b]$,

2. $f$ is $\alpha$-Generalized fractional differentiable on $(a, b)$ for some $\alpha \in(0,1]$.

Then, there exists $c \in(a, b)$ such that $D^{\alpha} f(c)=\frac{\alpha w_{t, \alpha}(f(b)-f(a))}{b-a}$.

Proof. Consider function

$$
h(t)=f(t)-f(a)-\frac{\alpha w_{t, \alpha}(f(b)-f(a))}{b-a}\left(\frac{t^{\alpha}}{\alpha w_{t, \alpha}}-\frac{a^{\alpha}}{\alpha w_{t, \alpha}}\right) .
$$

Then, the function $h$ satisfies the conditions of the fractional Rolle's Theorem. Hence, there exists $c \in(a, b)$ such that $D^{\alpha} h(c)=0$. We have the result since

$$
D^{\alpha} h(c)=D^{\alpha} f(c)-\frac{\alpha w_{t, \alpha}(f(b)-f(a))}{b-a}(1)=0 .
$$




\section{Generalized Partial Fractional Derivative}

In this section we introduce a partial fractional derivative of first and second order. Also we introduce a partial fractional differential ring.

Definition 4. Let $f\left(t_{1}, \cdots, t_{n}\right):[0, \infty)^{n} \rightarrow \mathbb{R}$ be a function with $n$ variables such that $\forall i$, then there exists the partial derivative of $f$ respect to $t_{i}$. Let $\alpha \in(0,1]$ be a fractional number. We define $\alpha$-generalized partial fractional derivative(GPFD) of $f$ with respect to $t_{i}$ at point $t=\left(t_{1}, \ldots, t_{n}\right)$

$$
\frac{\partial^{\alpha} f(t)}{\partial t_{i}^{\alpha}}=\lim _{\varepsilon \rightarrow 0} \frac{f\left(t_{1}, \ldots, t_{i}+w_{t_{i}, \alpha} t_{i}^{1-\alpha} \mathcal{\varepsilon}, \ldots, t_{n}\right)-f(t)}{\varepsilon},
$$

where $w_{t_{i}, \alpha}$ can be a function depend on $\alpha$ and $t_{i}$.

Remark 2. As a consequence of Definition 2 we can see for $\alpha \in(0,1]$ and $1 \leq i \leq n$ :

$$
\frac{\partial^{\alpha} f}{\partial t_{i}^{\alpha}}(t)=w_{t_{i}, \alpha}\left(t_{i}\right)^{1-\alpha} \frac{\partial f}{\partial t_{i}}(t)
$$

Let $\alpha \in(0,1]$ and $1 \leq i \leq n$. A partial differentiable function $f:[0, \infty)^{n} \rightarrow \mathbb{R}$ is said to be a $\alpha$-generalized partial fractional differentiable function respect to $t_{i}$ over $[0, \infty)$ if there exists $\frac{\partial^{\alpha} f(t)}{\partial t_{i}^{\alpha}}$ for all $t \in[0, \infty)$. We denote by $C_{i}^{\alpha}[0, \infty)^{n}$ the set of $\alpha$-generalized partial fractional differentiable functions respect to $t_{i}$ with real values in the interval $[0, \infty)^{n}$ in variable $t=\left(t_{1}, \ldots, t_{n}\right)$. The set $\left(C_{i}^{\alpha}[0, \infty)^{n},+,.\right)$ is a ring.

Theorem 5. Let $\alpha \in(0,1]$ and $1 \leq i \leq n$. The ring $C_{i}^{\alpha}[0, \infty)^{n}$ with operator

$$
\frac{\partial^{\alpha}}{\partial t_{i}^{\alpha}}: C_{i}^{\alpha}[0, \infty)^{n} \rightarrow C_{i}^{\alpha}[0, \infty)^{n}
$$

is a differential ring.

Proof. Since the ring $C_{i}^{\alpha}[0, \infty)^{n}$ is a commutative ring with unity $f\left(t_{1}, \ldots, t_{n}\right)=1$ and the derivation $\frac{\partial^{\alpha}}{\partial t_{i}^{\alpha}}$ for $\alpha \in(0,1]$ satisfies the following properties from Remark 2;

1. $\frac{\partial^{\alpha}\left(f_{1}+f_{2}\right)}{\partial t_{i}^{\alpha}}=\frac{\partial^{\alpha}\left(f_{1}\right)}{\partial t_{i}^{\alpha}}+\frac{\partial^{\alpha}\left(f_{2}\right)}{\partial t_{i}^{\alpha}} \quad f_{1}, f_{2} \in C^{\alpha}[0, \infty)^{n}$,

2. $\frac{\partial^{\alpha}\left(f_{1} f_{2}\right)}{\partial t_{i}^{\alpha}}=f_{1} \frac{\partial^{\alpha} f_{2}}{\partial t_{i}^{\alpha}}+f_{2} \frac{\partial^{\alpha^{i}} f_{1}}{\partial t_{i}^{\alpha}} \quad f_{1}, f_{2} \in C^{\alpha}[0, \infty)^{n}$.

Let $\alpha \in(0,1]$ and $1 \leq i \leq n$. Further let $f_{1}, f_{2} \in C^{\alpha}[0, \infty)^{n}$ be two functions, then GPFD has the following properties from Remark 2:

1. $\frac{\partial^{\alpha}\left(\frac{f_{1}}{f_{2}}\right)}{\partial t_{i}^{\alpha}}=\frac{f_{2} \frac{\partial^{\alpha} f_{1}}{\partial t_{i}^{\alpha}}-f_{1} \frac{\partial^{\alpha} f_{2}}{\partial t_{i}^{\alpha}}}{f_{2}^{2}}$,

2. $\frac{\partial^{\alpha} f_{1} \circ f_{2}}{\partial t_{i}^{\alpha}}=\frac{t_{i}^{\alpha-1}}{w_{t_{i}, \alpha}} \frac{\partial^{\alpha}\left(f_{1}\left(f_{2}\right)\right)}{\partial t_{i}^{\alpha}} \frac{\partial^{\alpha}\left(f_{2}\right)}{\partial t_{i}^{\alpha}}$,

3. $\frac{\partial^{\alpha+\beta}\left(f_{1}\right)}{\partial t_{i}^{\alpha}}=\frac{w_{t_{i}, \alpha} w_{t_{i}, \beta_{i}}}{w_{t_{i}, \alpha+\beta}} \frac{\partial^{\alpha}}{\partial t_{i}^{\alpha}} \frac{\partial^{\alpha}\left(f_{1}\right)}{\partial t_{i}^{\alpha}}$.

By considering previous properties of GPFD we called the ring $C_{i}^{\alpha}[0, \infty)^{n}$ a $w_{t_{i}, \alpha}$ - generalized partial fractional differential ring. We denote it by $\left(C_{i}^{\alpha}[0, \infty)^{n}, \frac{\partial^{\alpha}}{\partial t_{i}^{\alpha}}, w_{t_{i}, \alpha}\right)$.

We can see the following result by using the previous properties:

Theorem 6. Let $\alpha \in(0,1]$ and $1 \leq i \leq n$. Associated to any $\alpha$ and any $w_{t_{i}, \alpha}$ there is a partial fractional differential ring.

Example 3. Let $f\left(t_{1}, t_{2}\right)=t_{1}^{3} \sin \left(t_{2}\right)$ and $\alpha \in[0,1)$ then we have

$$
\frac{\partial^{\alpha} f}{\partial t_{1}^{\alpha}}=w_{t, \alpha}\left(t_{1}\right)^{1-\alpha}\left(3 t_{1}^{2}\right) \sin \left(t_{2}\right) .
$$


Definition 5. Let $\alpha \in(0,1]$ be a fractional number. We define $\alpha$-generalized partial fractional derivative of second order with respect to $t_{i}$ and $t_{j}$ at point $t=\left(t_{1}, \cdots, t_{n}\right)$ is

$$
\frac{\partial^{2} f(t)}{\partial t_{j}^{\alpha} \partial t_{i}^{\alpha}}=\frac{\partial^{\alpha}}{\partial t_{j}^{\alpha}}\left(\frac{\partial^{\alpha} f(t)}{\partial t_{i}^{\alpha}}\right)=\lim _{\varepsilon} \rightarrow 0 \frac{\frac{\partial^{\alpha} f}{\partial t_{i}^{\alpha}}\left(t_{1}, \ldots, t_{j}+w_{t_{j}, \alpha} t_{j}^{1-\alpha} \varepsilon, \ldots, t_{n}\right)-\frac{\partial^{\alpha} f(t)}{\partial t_{i}^{\alpha}}}{\varepsilon} .
$$

Remark 3. As a consequence of Definition 5 we can see for $\alpha \in(0,1]$ and $1 \leq i, j \leq n$;

$$
\frac{\partial^{\alpha^{2}} f(t)}{\partial t_{j}^{\alpha} \partial t_{i}^{\alpha}}=w_{t_{j}, \alpha} w_{t_{i}, \alpha}\left(t_{j} t_{i}\right)^{1-\alpha} \frac{\partial}{\partial t_{j}}\left(\frac{\partial f}{\partial t_{i}}(t)\right) .
$$

A partial differentiable function of second order $f:[0, \infty)^{n} \rightarrow \mathbb{R}$ is said to be a $\alpha$-generalized fractional partial differentiable function of second order respect to $t_{i}$ and $t_{j}$ over $[0, \infty)$ if there exists $\frac{\partial^{\alpha^{2}} f(t)}{\partial t_{j}^{\alpha} \partial t_{i}^{\alpha}}$ for all $t \in$ $[0, \infty)$. We denote $C_{i, j}^{\alpha^{2}}[0, \infty)^{n}$ the set of $\alpha$-generalized partial fractional differentiable functions of second order respect to $t_{i}$ and $t_{j}$ with real values in the interval $[0, \infty)^{n}$ in variable $t=\left(t_{1}, \ldots, t_{n}\right)$. The set $\left(C_{i, j}^{\alpha^{2}}[0, \infty)^{n},+,.\right)$ is a ring.

\section{Generalized Fractional Derivative for $\alpha \in(n, n+1]$}

In this section we define a fraction differential derivative for $\alpha \in(n, n+1]$.

Definition 6. Let $\alpha \in(n, n+1]$ be a fractional number for $n \in \mathbb{N}$ and $f:[0 . \infty) \rightarrow \mathbb{R}$ be a $n$ - differentiable. The generalized fractional derivative of order $\alpha$ is defined by

$$
\left.D^{\alpha} f(t)=\lim _{\varepsilon \rightarrow 0} \frac{f^{[\alpha]-1}\left(t+w_{t, \alpha}[\alpha]-\alpha\right.}{\varepsilon}\right)-f^{[\alpha]-1}(t),
$$

where $[\alpha]$ is the smallest integer greater than or equal to $\alpha$.

As a consequence of Definition 6 we can see

$$
D^{\alpha}(f)=w_{t, \alpha} t^{[\alpha]-\alpha} D^{[\alpha]}(f),
$$

where $\alpha \in(n, n+1]$.

Let $n<\alpha \leq n+1$. A function $f:[0, \infty) \rightarrow \mathbb{R}$ is said to be $\alpha$-generalized differentiable over $[0, \infty)$ if there exists $D^{\alpha}(f)(t)$ for all $t \in[0, \infty)$. We denote $C^{\alpha}[0, \infty)$ the set of $\alpha$-generalized fractional differentiable functions with real values in the interval $[0, \infty)$ in variable $t$. The set $\left(C^{\alpha}[0, \infty),+,.\right)$ is a ring.

Theorem 7. The ring $\left(C^{\alpha}[0, \infty),+..\right)$ with operator $D^{\alpha}$ is not a differential ring for fractional number $\alpha \in(n, n+1]$.

Proof. Since $D^{\alpha}(f g) \neq f D^{\alpha} g+g D^{\alpha}(f)$ for every $f, g \in C^{\alpha}[0, \infty)$.

\section{7. $\alpha$-Fractional Taylor Series}

There are some articles about fractional Taylor series see ([12,27-29]). In this section we use GFD to define a fractional taylor series for a function $f \in C^{r}[0, \infty)$ for every fractional number $r$.

Let $0<\alpha<1$, we define the $\alpha$-fractional taylor series of $f$ at real number $x_{0}$

$$
f(x)=f\left(x_{0}\right)+\sum_{i=1}^{\infty} \frac{D^{i} f\left(x_{0}\right)}{w_{x, \alpha} \overline{(\alpha+i-1) !}}\left(x-x_{0}\right)^{\alpha+i-1},
$$

where $\overline{(\alpha+i-1) !}=\alpha(\alpha+1) \cdots(\alpha+i-1)$.

Let $1<\alpha \leq 2$, we define the $\alpha$-fractional taylor series of $f$ at real number $x_{0}$

$$
f(x)=f\left(x_{0}\right)+D f\left(x_{0}\right)\left(x-x_{0}\right)+\sum_{i=2}^{\infty} \frac{D^{i} f\left(x_{0}\right)\left(x-x_{0}\right)^{\alpha+i-2}}{w_{x, \alpha} \overline{(\alpha+i-2) !}},
$$

where $\overline{(\alpha+i-2) !}=(\alpha-1) \alpha(\alpha+1) \cdots(\alpha+i-2)$. 
Let $n<\alpha \leq n+1$ such that $\alpha=n+A$ with $0<A<1$. We define the $\alpha$-fractional taylor series of $f$ at real number $x_{0}$,

$$
f(x)=f\left(x_{0}\right)+\sum_{i=1}^{n} \frac{D^{i} f\left(x_{0}\right)\left(x-x_{0}\right)^{i}}{i !}+\sum_{i=n+1}^{\infty} \frac{D^{i} f\left(x_{0}\right)}{w_{x, \alpha} \overline{(A+i-1) !}}\left(x-x_{0}\right)^{A+i-1},
$$

where $\alpha=n+A, \overline{(A+i-1) !}=A(A+1) \cdots(A+i-1)$.

\section{Application to differential equations}

There are some articles for applications of fractional differential derivative such as $[3,6,7,9]$. In this section we solve some (partial) fractional differential equations by using our definitions. Firstly we solve the fractional differential equations with the form

$$
a D^{\alpha} y+b y=c,
$$

where $y=f(t)$ be a differentiable function and $0<\alpha<1$.

By substituting GFD in the Equation (7) we have

$$
a w_{t, \alpha} t^{1-\alpha} D y+b y=c \Longrightarrow D y+\frac{b t^{\alpha-1}}{a w_{t, \alpha}} y=\frac{t^{\alpha-1} c}{a w_{t, \alpha}}
$$

the solutions of this equation have the form $y(t)=\frac{c}{b}+c_{1} e^{\left(-\frac{b t^{\alpha}}{a w t_{, \alpha} \alpha^{\alpha}}\right)}$.

Example 4. We consider the partial fractional differential equation with boundary conditions

$$
\left\{\begin{array}{l}
u_{t}+2 \sqrt[3]{x u_{x}}+u=x^{2}, t>0 \\
u(t, 0)=0 \\
u(0, x)=0
\end{array}\right.
$$

where $u(x, t)$ be a differentiable function respect to $x$ and $t, u(x, t)$ be a $\frac{1}{3}$-partial fractional differentiable function of first order respect to $x, u_{t}=\frac{\partial u}{\partial t}$ and $\sqrt[3]{u_{x}}=\frac{\partial^{\frac{1}{3}} u}{\partial x^{\frac{1}{3}}}$. For $w_{t, \frac{1}{3}}=\frac{1}{3}$, by using Remark 2 we can write

$$
u_{t}+2 \sqrt[3]{x u_{x}}+u=x^{2} \Longrightarrow u_{t}+2 \sqrt[3]{x} w_{t, \alpha} x^{1-\alpha} u_{x}+u=x^{2} \Longrightarrow u_{t}+\frac{2}{3} x u_{x}+u=x^{2}
$$

We solve this equation by taking Laplace transform of equation with respect to $t$. We denote by $U(x, s)$ the Laplace of $u(x, t)$ with respect to $t$, we have the following equation

$$
\left\{\begin{array}{l}
s U(x, s)-U(x, 0)+\frac{2}{3} x U_{x}(x, s)+U(x, s)=x^{2} s \\
U(t, 0)=0 \\
U(0, x)=0 .
\end{array}\right.
$$

Then

$$
U_{x}+\frac{3+3 s}{2 x} U=\frac{3 x}{2 s} \Longrightarrow U(x, s)=\frac{3 x^{2}}{s(3 s+7)}+c(s) x^{\frac{-3-3 s}{2}} .
$$

By substituting $U(0, x)=0$, we have $c(s)=0$, then $U(x, s)=x^{2}\left(\frac{3}{7 s}+\frac{9}{7(3 s+7)}\right)$. The solution of equation is $u(x, t)=\frac{x^{2}}{7}\left(1-e^{\frac{-7}{3}}\right)$.

Example 5. We consider the partial fractional differential equation of second order;

$$
\sqrt[5]{u_{x t}}+2 \frac{u}{x}=0
$$

where $u(x, t)$ be a $\frac{1}{5}$-fractional partial differentiable function of second order respect to $t, x$. For $w_{x, \frac{1}{5}}=$ $x^{2}, w_{t, \frac{1}{5}}=\frac{1}{\sqrt[3]{t}}$ by using remark 3 , we have

$$
x^{2} \sqrt[5]{x^{4}} \sqrt[5]{t^{4}} u_{x t}+2 \sqrt[3]{t} u=0
$$


We consider a solution of this differential equation of the form $u(x, t)=f(x) g(t)$ such that $f$ a function depends on $x$ and $g$ a function depends on $t$. By substituting $u(x, t)$ in the Equation (11) we have

$$
x^{2} \sqrt[5]{x^{4}} \sqrt[5]{t^{4}} D f \cdot D g+3 \sqrt[3]{t} f g=0
$$

We can write Equation (12) in a form that divide the functions of $t$ and $x$ :

$$
\frac{x^{2} \sqrt[5]{x^{4}} D f}{f}=-\frac{2 \sqrt[3]{t} g}{\sqrt[5]{t^{4}} D g}
$$

two sides of the Equality (13) is a constant $k$. We have

$$
\left\{\begin{array}{l}
\frac{D f}{f}=\frac{k}{\sqrt[5]{x^{14}}} \rightarrow \operatorname{Lnf}=k \sqrt[15]{x}+c_{1} \rightarrow f=\exp \left(k \sqrt[15]{x}+c_{1}\right), \\
\frac{D g}{g}=\frac{-2}{k \sqrt[15]{t^{7}}} \rightarrow \operatorname{Lng}=\frac{-2 \sqrt[15]{t^{8}}}{k}+c_{2} \rightarrow g=\exp \left(\frac{-2 \sqrt[15]{t^{8}}}{k}+c_{2}\right) .
\end{array}\right.
$$

The solution of Equation (10) has the form

$$
u(x, t)=\exp \left(k \sqrt[15]{x}+c_{1}\right) \exp \left(\frac{-2 \sqrt[15]{t^{8}}}{k}+c_{2}\right)=c \exp \left(k \sqrt[15]{x}+\frac{-2 \sqrt[15]{t^{8}}}{k}\right)
$$

\section{Conclusion}

We defined a generalized fractional derivative (GFD). We showed that the previous derivatives are particular cases. We also showed how it is possible to have infinite fractional derivatives with their algebra. We present the fractional differential ring, the fractional partial derivatives and their applications.

Conflicts of Interest: The authors declare no conflict of interest.

Data Availability: No data is required for this research.

Funding Information: No funding is available for this research.

Acknowledgments: This paper was supported by PRODEP of Mexico for Zeinab Toghani postdoctoral position.

\section{References}

[1] Laskin, N. (2002). Fractional schródinger equation. Physical Review E, 66(5), 056108, https://doi.org/10.1103/PhysRevE.66.056108.

[2] Wei, Y. (2015). Some solutions to the fractional and relativistic Schrödinger equations. International Journal of Theoretical and Mathematical Physics, 5, 87-111.

[3] Agarwal, P. (2012). On applications of fractional calculus involving summations of series. American Journal of Mathematics and Statistics, 2(6), 221-225.

[4] Du, M., Wang, Z., \& Hu, H. (2013). Measuring memory with the order of fractional derivative. Scientific Reports, 3, 3431, https://doi.org/10.1038/srep03431.

[5] Cenesiz, Y., \& Kurt, A. (2015). The solutions of time and space conformable fractional heat equations with conformable Fourier transform. Acta Universitatis Sapientiae, Mathematica, 7(2), 130-140.

[6] Wang, X., Zhang, F., \& Ding, J. (2017). Evaluation of water quality based on a machine learning algorithm and water quality index for the Ebinur Lake Watershed, China. Scientific Reports, 7, 12858, https://doi.org/10.1038/s41598-017-12853-y.

[7] Abu Hammad, I., \& Khalil, R. (2014). Fractional Fourier series with applications. American Journal of Computational and Applied Mathematics, 4(6), 187-191.

[8] Namias, V. (1980). The fractional order Fourier transform and its application to quantum mechanics. IMA Journal of Applied Mathematics, 25(3), 241-265.

[9] Younis, M., Zafar, A., Ul-Haq, K., \& Rahman, M. (2013). Travelling wave solutions of fractional order coupled Burgers' Equations by (G'/G)-expansion method. American Journal of Computational and Applied Mathematics, 3(2), 81-85.

[10] Anderson, D. R. (2016). Taylor's formula and integral inequalities for conformable fractional derivatives. In Contributions in Mathematics and Engineering (pp. 25-43). Springer, Cham.

[11] Khalil, R., Al Horani, M., Yousef, A., \& Sababheh, M. (2014). A new definition of fractional derivative. Journal of Computational and Applied Mathematics, 264, 65-70.

[12] Anderson, D. R., \& Ulness, D. J. (2015). Properties of the Katugampola fractional derivative with potential application in quantum mechanics. Journal of Mathematical Physics, 56(6), 063502, https://doi.org/10.1063/1.4922018. 
[13] Sousa, J. V. D. C., \& de Oliveira, E. C. (2017). On the local M-derivative. arXiv preprint arXiv:1704.08186.

[14] Camrud, E. (2016). The conformable ratio derivative. Rose-Hulman Undergraduate Mathematics Journal, 17(2), Article 10. Available at: https://scholar.rose-hulman.edu/rhumj/vol17/iss2/10.

[15] Miller, K. S., \& Ross, B. (1993). An introduction to the fractional calculus and fractional differential equations. Wiley.

[16] Manuel, D., \& Machado, J. T. (2015). What is a fractional derivative. Journal of Computational Physics, 239, 4-13.

[17] Katugampola, U. N. (2014). A new fractional derivative with classical properties. arXiv preprint arXiv:1410.6535.

[18] Iyiola, O. S., \& Nwaeze, E. R. (2016). Some new results on the new conformable fractional calculus with application using D'Alambert approach. Progress in Fractional Differentiation and Applications, 2(2), 115-122.

[19] Abdeljawad, T., AL Horani, M., \& Khalil, R. (2015). Conformable fractional semigroups of operators. Journal of Semigroup Theory and Applications, 2015, Article-ID 7. Available at: http://scik.org/index.php/jsta/article/view/2410.

[20] Guebbai, H., \& Ghiat, M. (2016). New conformable fractional derivative definition for positive and increasing functions and its generalization. Advances in Dynamical Systems and Applications, 11(2), 105-111.

[21] Sarikaya, M. Z., \& Budak, H. (2017). New inequalities of Opial type for conformable fractional integrals. Turkish Journal of Mathematics, 41(5), 1164-1173.

[22] Caputo, M., \& Fabrizio, M. (2015). A new definition of fractional derivative without singular kernel. Progress in Fractional Differentiation and Applications, 1(2), 1-13.

[23] Laskin, N. (2000). Fractional quantum mechanics. Physical Review E, 62(3), 3135, https://doi.org/10.1103/PhysRevE. 62.3135.

[24] Caputo, M. (1969). Elasticita de dissipazione, Zanichelli, Bologna, Italy,(Links).

[25] Anderson, D. R., \& Ulness, D. J. (2015). Newly defined conformable derivatives. Advances in Dynamical Systems and Applications, 10(2), 109-137.

[26] van der Waerden, B. L. (1950). Joseph Fels Ritt, Differential algebra. Bulletin of the American Mathematical Society, 56(6), 521-523.

[27] Usero, D. (2008). Fractional Taylor series for Caputo fractional derivatives. Construction of numerical schemes. Available at: http://www.fdi.ucm. es/profesor/lvazquez/calcfrac/docs/paper_Usero. pdf.

[28] Munkhammar, J. (2004). Riemann-Liouville fractional derivatives and the Taylor-Riemann series. Department of Mathematics Uppsala University, U.U.D.M. Project Report 2004:7. Available at: https:/ /www.diva-portal.org/smash /get/diva2:305264/FULLTEXT01.pdf.

[29] Yang, X. J. (2011). Generalized local fractional Taylor's formula with local fractional derivative. arXiv preprint arXiv:1106.2459.

(C) 2021 by the authors; licensee PSRP, Lahore, Pakistan. This article is an open access article distributed under the terms and conditions of the Creative Commons Attribution (CC-BY) license (http://creativecommons.org/licenses/by/4.0/). 